\title{
AN EXACT ASYMPTOTICS FOR THE MOMENT OF CROSSING A CURVED BOUNDARY BY AN ASYMPTOTICALLY STABLE RANDOM WALK*
}

\author{
V. I. WACHTEL ${ }^{\dagger}$ AND D. E. DENISOV $\ddagger$ \\ (Translated by the authors)
}

\begin{abstract}
Suppose that $\left\{S_{n}, n \geqq 0\right\}$ is an asymptotically stable random walk. Let $g$ be a positive function and $T_{g}$ be the first time when $S_{n}$ leaves $[-g(n), \infty)$. In this paper we study asymptotic behavior of $T_{g}$. We provide integral tests for function $g$ that guarantee $\mathbf{P}\left(T_{g}>n\right) \sim$ $V(g) \mathbf{P}\left(T_{0}>n\right)$, where $T_{0}$ is the first strict descending ladder epoch of $\left\{S_{n}\right\}$.
\end{abstract}

Key words. first passage time, moving boundary, nonlinear boundary, exit time

DOI. $10.1137 /$ S0040585X97T987740

1. Introduction and main results. Consider a one-dimensional random walk

$$
S_{0}=0, \quad S_{n}=X_{1}+\cdots+X_{n}, \quad n \geqq 1,
$$

where $X, X_{1}, X_{2}, \ldots$ are independent and identically distributed random variables. Let $g(t)$ be an increasing function and consider the exit time from the domain bounded by $-g(t)$, that is,

$$
T_{g}:=\min \left\{n \geqq 1: S_{n}<-g(n)\right\} .
$$

The aim of this paper is to study the asymptotics $\mathbf{P}\left(T_{g}>n\right)$ as $n$ goes to infinity.

This question has been thoroughly studied in the case of constant boundary, that is, for $g(n) \equiv x \geqq 0$. In particular, it is well known that if

$$
\mathbf{P}\left(S_{n} \geqq 0\right) \rightarrow \rho \in(0,1), \quad n \rightarrow \infty,
$$

then

$$
\mathbf{P}\left(T_{x}>n\right) \sim h(x) n^{\rho-1} L(n), \quad n \rightarrow \infty,
$$

where $L(n)$ is a slowly varying function. (Slightly abusing notation, we write $T_{x}$ instead of $T_{g}$ when $g(t) \equiv x$. We also write $a(x) \sim b(x)$ if $a(x) / b(x) \rightarrow 1$ as $x$ tends to infinity.) Function $h(x)$ is the renewal function of the strict decreasing ladder height process.

We now introduce a class of random walks that we will considered in the present paper. Let

$$
\begin{aligned}
\mathcal{A}:= & \{0<\alpha<1 ;|\beta|<1\} \cup\{1<\alpha<2 ;|\beta| \leqq 1\} \\
& \cup\{\alpha=1, \beta=0\} \cup\{\alpha=2, \beta=0\}
\end{aligned}
$$

\footnotetext{
* Received by the editors June 3, 2014. Originally published in the Russian journal Teoriya Veroyatnostei $i$ ee Primeneniya, 60 (2015), No. 3, pp. 459-481.

http://www.siam.org/journals/tvp/60-3/T98774.html

${ }^{\dagger}$ Institut für Mathematik, Universität Augsburg, Augsburg, Germany (Vitali.Wachtel@math.uniaugsburg.de).

${ }^{\ddagger}$ School of Mathematics, University of Manchester, Manchester, UK (denis.denisov@ manchester.ac.uk).
} 
be a subset of $\mathbf{R}^{2}$. For $(\alpha, \beta) \in \mathcal{A}$ and a random variable $X$ we write $X \in \mathcal{D}(\alpha, \beta)$, if the distribution of $X$ belongs to the domain of attraction of the stable law with characteristic function

$$
G_{\alpha, \beta}(t):=\exp \left\{-c|t|^{\alpha}\left(1-i \beta \frac{t}{|t|} \operatorname{tg} \frac{\pi \alpha}{2}\right)\right\}, \quad c>0,
$$

and, in addition, $\mathbf{E} X=0$, if the first moment exists. Let $c(x)$ be a positive function specified by the relation

$$
c(x):=\inf \left\{u \geqq 0: \mu(u) \leqq x^{-1}\right\}, \quad x \geqq 1,
$$

where

$$
\mu(u):=\frac{1}{u^{2}} \int_{-u}^{u} x^{2} \mathbf{P}(X \in d x) .
$$

It is known (see, for instance, [7, Chap. XVII, section 5]) that for every $X \in \mathcal{D}(\alpha, \beta)$ the function $\mu(u)$ is regularly varying with index $(-\alpha)$. This implies that $c(x)$ is regularly varying with index $\alpha^{-1}$, i.e., there exists a function $l_{1}(x)$, slowly varying at infinity, such that

$$
c(x)=x^{1 / \alpha} l_{1}(x) .
$$

In addition, the scaled sequence $\left\{S_{n} / c(n), n \geqq 1\right\}$ converges in distribution to the stable law given by (3). In this case we say that $S_{n}$ is an asymptotically stable random walk. For every $X \in \mathcal{D}(\alpha, \beta)$ there is an explicit formula for $\rho$,

$$
\rho= \begin{cases}\frac{1}{2}, & \alpha=1, \\ \frac{1}{2}+\frac{1}{\pi \alpha} \operatorname{arctg}\left(\beta \operatorname{tg} \frac{\pi \alpha}{2}\right) & \text { otherwise. }\end{cases}
$$

Since $h(0)=1$, one can rewrite (2) slightly differently:

$$
\mathbf{P}\left(T_{x}>n\right) \sim h(x) \mathbf{P}\left(T_{0}>n\right) .
$$

This representation shows that the asymptotics are the same up to a constant for every fixed $x$. This statement remains valid for curved boundaries which do not grow too fast. For the Brownian motion and monotonically increasing function $g$ it was shown in [12] and [14] that

$$
0<\mathbf{E}\left|B\left(T_{g}^{(\mathrm{bm})}\right)\right|<\infty \quad \Longleftrightarrow \quad \int_{1}^{\infty} g(t) t^{-3 / 2} d t<\infty
$$

and, moreover,

$$
\mathbf{P}\left(T_{g}^{(\mathrm{bm})}>t\right) \sim \mathbf{E}\left[B\left(T_{g}^{(\mathrm{bm})}\right)\right] \mathbf{P}\left(T_{1}^{(\mathrm{bm})}>t\right) \quad \text { as } t \rightarrow \infty .
$$

Here, $T_{g}^{(\mathrm{bm})}$ denotes the corresponding exit time for the Brownian motion.

Finiteness of the integral in (7) is also necessary: if $\int_{1}^{\infty} g(t) t^{-3 / 2} d t=\infty$, then

$$
\mathbf{P}\left\{T_{g}^{(\mathrm{bm})}>t\right\} \gg \mathbf{P}\left\{T_{1}^{(\mathrm{bm})}>t\right\} \quad \text { as } t \rightarrow \infty .
$$

Copyright (c) by SIAM. Unauthorized reproduction of this article is prohibited. 
The case of general random walks and Lévy processes was analyzed by Greenwood and Novikov in [9] (see also [12], [13]). In Theorem 1 they state that if $\mathbf{E} g\left(T_{0}\right)<\infty$, then there exists $R_{g} \in(0, \infty)$ such that

$$
\mathbf{P}\left(T_{g}>n\right) \sim R_{g} \mathbf{P}\left(T_{0}>n\right) \quad \text { as } n \rightarrow \infty .
$$

However, recent results by Aurzada and Kramm [2] give strong grounds to suspect that conditions in [9] are not optimal. More precisely, it is shown in [2] that if $g(t)=o\left(t^{\gamma}\right)$ with some $\gamma<1 / \alpha$, then $\mathbf{P}\left(T_{g}>n\right)$ and $\mathbf{P}\left(T_{0}>n\right)$ have the same rough asymptotics, that is,

$$
\mathbf{P}\left(T_{g}>n\right)=n^{\rho-1+o(1)} \quad \text { as } n \rightarrow \infty .
$$

However, in view of (2), $\mathbf{E} g\left(T_{0}\right)$ is finite only for $\gamma \leqq 1-\rho<1 / \alpha$.

In this paper we present alternative (and milder than $\mathbf{E} g\left(T_{0}\right)<\infty$ ) conditions for the validity of (9).

THEOREM 1. Let $g$ be an increasing function such that $h(g(x))$ is subadditive. If (1) holds and

$$
\mathbf{E} h\left(g\left(T_{0}\right)\right)<\infty,
$$

then there exists a constant $V(g) \in(0, \infty)$ such that

$$
\mathbf{P}\left(T_{g}>n\right) \sim V(g) \mathbf{P}\left(T_{0}>n\right) \sim V(g) n^{\rho-1} L(n), \quad n \rightarrow \infty .
$$

This result generalizes Theorem 1 in [9], where it was assumed that $g$ is concave and $\mathbf{E} g\left(T_{0}\right)$ is finite. Noting that $h$ is subadditive, we conclude that (10) is weaker than the finiteness of $\mathbf{E} g\left(T_{0}\right)$. Moreover, subadditivity of $h$ implies also that $h(g(x))$ is subadditive for every concave function $g$. The subadditivity assumption can be further weakened.

Remark 1 . The statement of Theorem 1 remains valid if we replace subadditivity of $h(g(x))$ by the existence of a subadditive majorant which satisfies (10). walks.

Further we reformulate Theorem 1 to the case of asymptotically stable random

Corollary 1. Suppose that $X \in \mathcal{D}(\alpha, \beta)$. If

$$
\int_{1}^{\infty} \frac{h(g(x))}{x h(c(x))} d x<\infty
$$

then (11) holds.

If $\mathbf{E}\left(X^{-}\right)^{2}<\infty$, then $h(x) \sim c x$, and, consequently, our condition (12) coincides with Novikov's integral test; see Theorem 1 in [13]. Note also that Novikov [13] imposes a stronger assumption on $g$, namely, this function is assumed to be concave.

Since $h$ is regularly varying of index $\alpha(1-\rho)$, we conclude that (11) holds for every function $g$, bounded from above by $x^{\gamma}$ with some $\gamma<\min \{1,1 / \alpha\}$.

Remark 2. Mogulskii and Pecherskii [11] derived the following factorization identity for $T_{g}$ : if $g$ is superadditive, i.e., $g(x+y) \geqq g(x)+g(y)$, then there exists a sequence of events $E_{n}$ such that

$$
\sum_{n=0}^{\infty} z^{n} \mathbf{P}\left(T_{g}>n\right)=\exp \left\{\sum_{n=1}^{\infty} \frac{z^{n}}{n} \mathbf{P}\left(E_{n}\right)\right\}
$$

Copyright $@$ by SIAM. Unauthorized reproduction of this article is prohibited. 
Moreover,

$$
E_{n} \subseteq\left\{S_{n} \geqq-g(n)\right\} \quad \text { for all } n \geqq 1 .
$$

Unfortunately, the structure of $E_{n}$ is highly nontrivial, and it is not clear how to determine the limit of $\mathbf{P}\left(E_{n}\right)$. However, using (14), one can obtain an asymptotically precise upper bound for $\mathbf{P}\left(T_{g}>n\right)$. Indeed, it is immediate from (14) that

$$
\mathbf{P}\left(T_{g}>n\right) \leqq q_{n},
$$

where $q_{n}$ is determined by

$$
\sum_{n=0}^{\infty} z^{n} q_{n}=\exp \left\{\sum_{n=1}^{\infty} \frac{z^{n}}{n} \mathbf{P}\left(S_{n} \geqq-g(n)\right)\right\} .
$$

Assume that function $g$ satisfies

$$
\int_{1}^{\infty} \frac{g(x)}{x c(x)} d x<\infty
$$

Then applying the estimate $\mathbf{P}\left(S_{n} \in[x, x+1)\right) \leqq C / c(n)$, we conclude that coefficients of

$$
R(z):=\exp \left\{\sum_{n=1}^{\infty} \frac{z^{n}}{n} \mathbf{P}\left(S_{n} \in[-g(n), 0]\right)\right\}
$$

are summable, i.e., $R(1)<\infty$. Noting now that

$$
\sum_{n=0}^{\infty} z^{n} q_{n}=\left(\sum_{n=0}^{\infty} z^{n} \mathbf{P}\left(T_{0}>n\right)\right) R(z)
$$

we arrive at the relation

$$
q_{n} \sim R(1) \mathbf{P}\left(T_{0}>n\right)
$$

and, consequently,

$$
1 \leqq \liminf _{n \rightarrow \infty} \frac{\mathbf{P}\left(T_{g}>n\right)}{\mathbf{P}\left(T_{0}>n\right)} \leqq \limsup _{n \rightarrow \infty} \frac{\mathbf{P}\left(T_{g}>n\right)}{\mathbf{P}\left(T_{0}>n\right)} \leqq R(1) .
$$

Note also that in order to obtain the relation $\mathbf{P}\left(T_{g}>n\right) \sim C \mathbf{P}\left(T_{0}>n\right)$ from (13) it suffices to show that

$$
\sum_{n=1}^{\infty} \frac{1}{n}\left|\mathbf{P}\left(E_{n}\right)-\mathbf{P}\left(S_{n}>0\right)\right|<\infty
$$

Recalling that $h$ is regularly varying of index $\alpha(1-\rho)$, we conclude that (12) is more restrictive than (15) for all random walks with $\alpha(1-\rho)<1$. Thus, bounds (16) imply that $\mathbf{P}\left(T_{g}>n\right)$ can be of the same order as $\mathbf{P}\left(T_{0}>n\right)$ for functions $g$ which do not satisfy (12).

The starting point of the proof of Theorem 1 is the following well-known observation: $T_{g}$ coincides with one of the strict descending ladder epochs of $S_{n}$. Let $\left(\tau_{k}, \chi_{k}\right)$ be independent copies of $\left(T_{0},-S_{T_{0}}\right)$. Then

$$
T_{g}=\sum_{k=1}^{\nu} \tau_{k},
$$

Copyright (c) by SIAM. Unauthorized reproduction of this article is prohibited. 
where

$$
\nu:=\min \left\{k \geqq 1: \chi_{1}+\cdots+\chi_{k}>g\left(\tau_{1}+\cdots+\tau_{k}\right)\right\} .
$$

Since the tail distribution function of $\tau$ 's is regularly varying with index $\rho-1 \in(-1,0)$, we shall prove that, for any increasing function $g$,

$$
\lim _{n \rightarrow \infty} \frac{\mathbf{P}\left(T_{g}>n\right)}{\mathbf{P}\left(T_{0}>n\right)}=\mathbf{E} \nu \in[1, \infty] .
$$

Thus, to prove Theorem 1 it suffices to show that $\mathbf{E} \nu$ is finite under the conditions of Theorem 1.

The subadditivity assumption in Theorem 1 seems to be purely technical. Unfortunately, we do not know how to remove this restriction. But for asymptotically stable random walks one can replace (12) by a stronger integral test which allows us to derive (11) without a further subadditivity assumption.

Theorem 2. Assume that $X \in \mathcal{D}(\alpha, \beta)$. If $g$ is increasing and

$$
\int_{1}^{\infty} \frac{h(g(x))}{x h(c(x / \log x))} d x<\infty
$$

then (11) remains valid.

Integral test (20) is fulfilled for any increasing regularly varying with index $\gamma<$ $1 / \alpha$ function $g$; consequently, the subadditivity assumption is superfluous for such functions. On the other hand, it is not difficult to construct a regularly varying with index $1 / \alpha$ function $g$ that satisfies (12), while the integral in (20) is infinite.

Our proof of Theorem 2 is based on an appropriate adaptation of the method from [4], where we studied the asymptotic behavior of exit times from cones of multidimensional random walks. The main idea in [4] was to use the classical universality for random walks: If certain moments of a random walk are finite (this means that random walk is sufficiently close to the Brownian motion), then the asymptotics for the exit time of this random walk is the same, up to a constant factor, as that for the Brownian motion. In the proof of Theorem 2 we use a completely different type of universality: We fix the distribution of a random walk and look for boundaries $g$ such that $T_{g}$ and $T_{0}$ have the same rate of decrease.

Now we turn to exit times from a shrinking domain. Let

$$
\widehat{T}_{g}:=\min \left\{n \geqq 1: S_{n}<g(n)\right\} .
$$

For $\widehat{T}_{g}$ one does not have any representation similar to (17). For that reason there is no analogue of Theorem 1 for $\widehat{T}_{g}$. But one can look at the ratio $\mathbf{P}\left(\widehat{T}_{g}>n\right) / \mathbf{P}\left(T_{0}>n\right)$ in the following way: If $g$ is positive, then

$$
\frac{\mathbf{P}\left(\widehat{T}_{g}>n\right)}{\mathbf{P}\left(T_{0}>n\right)}=\mathbf{P}\left(\widehat{T}_{g}>n \mid T_{0}>n\right)
$$

and one can try to represent the limit of this conditional probability as a functional of $\left\{S_{n}\right\}$ conditioned to stay nonnegative. To formulate the corresponding result we have to introduce some notation. It is well known that $h(x)$ is a positive harmonic function for $\left\{S_{n}\right\}$ killed at leaving $[0, \infty)$, i.e.,

$$
\mathbf{E}[h(x+X) ; x+X>0]=h(x), \quad x \geqq 0 .
$$


We denote by $\mathbf{P}^{h}$ the Doob transform of $\mathbf{P}$ by the function $h$. More precisely, $\mathbf{P}^{h}$ is the distribution of the Markov chain on $[0, \infty)$ with transition function

$$
p^{h}(x, d y)=\frac{h(y)}{h(x)} \mathbf{P}(x+X \in d y), \quad x, y \geqq 0 .
$$

The following statement is immediate from Lemma 2.5 in [1].

Proposition 1. If (1) holds, then

$$
\lim _{n \rightarrow \infty} \frac{\mathbf{P}\left(\widehat{T}_{g}>n\right)}{\mathbf{P}\left(T_{0}>n\right)}=\mathbf{P}^{h}\left(\widehat{T}_{g}=\infty\right) .
$$

Greenwood and Novikov [9, Theorem 2] have shown the existence of the limit in the left-hand side of (21) for random walks with $\mathbf{E} X=0$ and $\mathbf{E} e^{-\lambda X}<\infty$ for some $\lambda>0$. They have also shown that this limit is positive if and only if $\mathbf{E} g\left(T_{0}\right)<\infty$. The information about the positivity of this limit is very important, since if it is zero, then the asymptotic behavior of $\mathbf{P}\left(\widehat{T}_{g}>n\right)$ remains unknown. Thus, in order to apply Proposition 1 we need to find reasonable conditions for the positivity of $\mathbf{P}^{h}\left(\widehat{T}_{g}=\infty\right)$. Since $\mathbf{P}^{h}\left(\widehat{T}_{g}=\infty\right)=\mathbf{P}^{h}\left(S_{k} \geqq g(k)\right.$ for all $\left.k \geqq 1\right)$, we obtain that $\mathbf{P}^{h}\left(\widehat{T}_{g}=\infty\right)=1$ for any function $g$ such that $\mathbf{P}^{h}\left(S_{k}<g(k)\right.$ i.o. $)=0$. One has also the inverse implication: if $\mathbf{P}^{h}\left(S_{k}<g(k)\right.$ i.o. $)=1$, then $\mathbf{P}^{h}\left(\widehat{T}_{g}=\infty\right)=0$.

By [10, Theorem 1] if $\mathbf{E} X^{2}<\infty$ and $\mathbf{E} X=0$, then

$$
\begin{aligned}
& \mathbf{P}^{h}\left(S_{k}<g(k) \text { i.o. }\right)=0 \text { or } 1 \\
& \quad \text { if, respectively, the integral } \\
& \quad \int_{1}^{\infty} \frac{g(x)}{x^{3 / 2}} d x \text { is finite or infinite. }
\end{aligned}
$$

Consequently, for any oscillating random walk with finite variance,

$$
\mathbf{P}^{h}\left(\widehat{T}_{g}=\infty\right)>0 \quad \Longleftrightarrow \quad \int_{1}^{\infty} \frac{g(x)}{x^{3 / 2}} d x<\infty
$$

Unfortunately, we did not find results similar to (22) for random walks with infinite variance. Thus, we do not have any criterion for the positivity of $\mathbf{P}^{h}\left(\widehat{T}_{g}=\infty\right)$ for oscillating random walks with infinite variance.

It turns out that the approach used in Theorem 2 can also be applied to $\widehat{T}_{g}$.

Theorem 3. Assume that $X \in \mathcal{D}(\alpha, \beta)$ and that (20) holds. Then there exists $\widehat{V}(g) \in(0, \infty)$ such that, as $n \rightarrow \infty$,

$$
\mathbf{P}\left(\widehat{T}_{g}>n\right) \sim \widehat{V}(g) \mathbf{P}\left(T_{0}>n\right) .
$$

2. Proof of Theorem 1. We first derive (19). If $\mathbf{E} \nu$ is finite, then the desired relation follows immediately from Theorem 2 in [8]. Now assume that $\mathbf{E} \nu=\infty$. Since $\tau$ is positive,

$$
\mathbf{P}\left(T_{g}>n\right) \geqq \mathbf{P}\left(\sum_{k=1}^{\nu \wedge m} \tau_{k}>n\right)
$$


for any $m \geqq 1$. Applying Theorem 2 of [8] to the stopping time $\nu \wedge m$ we obtain

$$
\liminf _{n \rightarrow \infty} \frac{\mathbf{P}\left(T_{g}>n\right)}{\mathbf{P}\left(T_{0}>n\right)} \geqq \mathbf{E}[\nu \wedge m] .
$$

Letting $m$ go to infinity, we get the derivation of (19).

As it was already mentioned in the introduction, Theorem 1 follows from (19) and the following statement.

Proposition 2. Under the assumptions of Theorem 1

$$
\mathbf{E} \nu<\infty
$$

2.1. Proof of Proposition 2 for random walks with $\mathbf{E} \chi=\infty$. It follows from subadditivity of $h(g(x))$ that

$$
\nu \leqq \mu:=\min \left\{k \geqq 1: h\left(\chi_{1}+\cdots+\chi_{k}\right)>h\left(g\left(\tau_{1}\right)\right)+\cdots+h\left(g\left(\tau_{k}\right)\right)\right\} .
$$

Thus, it suffices to show that the expectation of $\mu$ is finite. But the latter is a consequence of the positive recurrence of zero for the sequence

$$
Z_{n}:=\left(Z_{0}+\sum_{k=1}^{n} h\left(g\left(\tau_{k}\right)\right)-h\left(\sum_{k=1}^{n} \chi_{k}\right)\right)^{+}, \quad n \geqq 1 .
$$

According to Lemma 1 and Proposition 1 in [6], our assumption $\mathbf{E} \chi=\infty$ is equivalent to $\mathbf{E} h(\chi)=\infty$. Consequently,

$$
\mathbf{E}[h(y+\chi)-h(y)]=\infty
$$

for all $y \geqq 0$. Combining this with (10), we finally obtain

$$
\mathbf{E}[h(y+\chi)-h(y)-h(g(\tau))]=\infty
$$

for all $y \geqq 0$.

Fix some positive $x$ and $y$ such that $x>h(y)$. Then

$$
\begin{aligned}
& \mathbf{E}\left[Z_{n+1}-Z_{n} \mid \sum_{k=1}^{n} h\left(g\left(\tau_{k}\right)\right)=x, \sum_{k=1}^{n} \chi_{k}=y\right] \\
& \quad \leqq \mathbf{E}[h(y)-h(y+\chi)+h(g(\tau)) ; x+h(g(\tau))-h(y+\chi)>0] \\
& \quad=-\mathbf{E}[h(y+\chi)-h(y)-h(g(\tau)) ; h(y+\chi)-h(y)-h(g(\tau))<x-h(y)] .
\end{aligned}
$$

Taking into account (25), we conclude that there exists $A>0$ such that

$$
\mathbf{E}\left[Z_{n+1}-Z_{n} \mid \sum_{k=1}^{n} h\left(g\left(\tau_{k}\right)\right)=x, \sum_{k=1}^{n} \chi_{k}=y\right] \leqq-1
$$

for all $x, y$, satisfying $x-h(y)>A$. This implies that the hitting time of $[0, A]$ by the sequence $Z_{n}$ has finite mean. Since 0 can be approached from any point of $[0, A]$ in a finite time, we conclude that $\mathbf{E} \mu<\infty$.

Copyright $@$ by SIAM. Unauthorized reproduction of this article is prohibited. 
2.2. Proof of Proposition 2 for random walks with $\mathbf{E} \chi<\infty$. If the random walk $S_{n}$ is such that $\mathbf{E} \chi<\infty$, then $h(x) \sim C x$ as $x \rightarrow \infty$ and, in particular, $\mathbf{E} h(\chi)<\infty$. Therefore, we cannot use the sequence $Z_{n}$ from the previous subsection. Fix some $k \geqq 1$ and consider

$$
Z^{(k)}:=\chi_{1}+\chi_{2}+\cdots+\chi_{k}-g\left(\tau_{1}+\tau_{2}+\cdots+\tau_{k}\right) ;
$$

let $Z_{i}^{(k)}$ be independent copies of $Z$. Using the subadditivity of $g$ once again, one can easily show that

$$
\nu \leqq k \mu^{(k)}
$$

where

$$
\mu^{(k)}:=\min \left\{n \geqq 1: Z_{1}^{(k)}+Z_{2}^{(k)}+\cdots+Z_{n}^{(k)}>0\right\} .
$$

From this we conclude that Proposition 2 follows from the existence of $k$ such that $\mathbf{E} Z^{(k)}>0$. Therefore, it suffices to show that

$$
\lim _{k \rightarrow \infty} \frac{1}{k} \mathbf{E} g\left(\tau_{1}+\tau_{2}+\cdots+\tau_{k}\right)=0 .
$$

Set $U_{k}=\tau_{1}+\tau_{2}+\cdots+\tau_{k}$ and note that $\tau_{i} \in \mathcal{D}(1-\rho, 1)$. Let $a(k)$ be the corresponding norming sequence, that is, $U_{k} / a(k)$ converges weakly towards a stable distribution of index $1-\rho$.

Since $g$ is increasing and sublinear,

$$
\begin{aligned}
\mathbf{E} g\left(U_{k}\right) & =\sum_{j=0}^{\infty} \mathbf{E}\left[g\left(U_{k}\right) ; U_{k} \in[j a(k),(j+1) a(k)]\right. \\
& \leqq g(a(k))+2 \sum_{j=1}^{\infty} g(j a(k)) \mathbf{P}\left(U_{k}>j a(k)\right)
\end{aligned}
$$

Since the tail of $\tau$ is regularly varying with index $\rho-1>-1$,

$$
\mathbf{P}\left(U_{k}>x\right) \leqq C k \mathbf{P}(\tau>x), \quad x \geqq 0 .
$$

Consequently,

$$
\begin{aligned}
\mathbf{E} g\left(U_{k}\right) & \leqq g(a(k))+C \sum_{j=1}^{\infty} g(j a(k)) k \mathbf{P}(\tau>j a(k)) \\
& \leqq g(a(k))+C k \mathbf{E}[g(\tau) ; \tau>a(k)] .
\end{aligned}
$$

It follows from the assumption $\mathbf{E} g(\tau)<\infty$ that

$$
\frac{g(a(k))}{k} \leqq C g(a(k)) \mathbf{P}(\tau>a(k)) \leqq C \mathbf{E}[g(\tau) ; \tau>a(k)] .
$$

Furthermore,

$$
\mathbf{E}[g(\tau) ; \tau>a(k)] \rightarrow 0 \quad \text { as } k \rightarrow \infty .
$$

Applying these relations to (27), we obtain (26). This completes the proof of Proposition 2 . 
2.3. Proof of Corollary 1. It suffices to show that (10) and (12) are equivalent. First, we note that if $X \in \mathcal{D}(\alpha, \beta)$, then

$$
\mathbf{P}\left(\tau_{1}>x\right) \sim C \frac{1}{h(c(x))} \quad \text { as } x \rightarrow \infty
$$

and, consequently, (12) is equivalent to

$$
\int_{1}^{\infty} \frac{h(g(x))}{x} \mathbf{P}\left(\tau_{1}>x\right) d x<\infty .
$$

Consequently, (12) is equivalent to $\mathbf{E} h\left(g\left(\tau_{1}\right)\right)<\infty$.

\section{Proof of Theorem 2.}

3.1. Finiteness of $\boldsymbol{V}(\boldsymbol{g})$. Let $h$ be the renewal function of the decreasing ladder height process. It is known that $h$ is harmonic for $S_{n}$ killed while leaving $\mathbf{R}_{+}$, that is,

$$
\mathbf{E}\left[h\left(x+X_{1}\right) ; x+X_{1}>0\right]=h(x), \quad x>0 .
$$

Extending $h(x)$ to the negative half line with 0 we can write this equality as $\mathbf{E} h(x+$ $\left.X_{1}\right)=h(x)$ for $x>0$.

Lemma 1. The sequence $Y_{n}=h\left(S_{n}+g(n)\right) 1\left\{T_{g}>n\right\}$ is a submartingale.

Proof. It is clear that

$$
\begin{aligned}
\mathbf{E}\left[Y_{n+1}-Y_{n} \mid \mathcal{F}_{n}\right]= & \mathbf{E}\left[\left(h\left(S_{n+1}+g(n+1)\right)-h\left(S_{n}+g(n)\right)\right) 1\left\{T_{g}>n\right\} \mid \mathcal{F}_{n}\right] \\
& -\mathbf{E}\left[h\left(S_{n+1}+g(n+1)\right) 1\left\{T_{g}=n+1\right\} \mid \mathcal{F}_{n}\right] .
\end{aligned}
$$

Now note that $h\left(S_{n+1}+g(n+1)\right) 1\left\{T_{g}=n+1\right\}=0$ since $h(x)=0$ for $x \leqq 0$. Further, by harmonicity of $h$ we obtain

$$
\begin{aligned}
& \mathbf{E}\left[Y_{n+1}-Y_{n} \mid \mathcal{F}_{n}\right] \\
& \quad=\mathbf{E}\left[\left(h\left(S_{n+1}+g(n+1)\right)\right) 1\left\{T_{g}>n\right\} \mid \mathcal{F}_{n}\right]-h\left(S_{n}+g(n)\right) 1\left\{T_{g}>n\right\} \\
& \quad=h\left(S_{n}+g(n+1)\right) 1\left\{T_{g}>n\right\}-h\left(S_{n}+g(n)\right) 1\left\{T_{g}>n\right\} \geqq 0,
\end{aligned}
$$

since $h$ and $g$ are monotonically increasing. Lemma 1 is proved.

Fix any positive sequence $\varepsilon_{n} \rightarrow 0$ and denote

$$
\nu_{n}:=\min \left\{k \geqq 1:\left|S_{k}\right| \geqq c\left(\varepsilon_{n} n\right)\right\} .
$$

Lemma 2. There exists a constant $C$ such that

$$
\mathbf{P}\left(\nu_{n}>\delta n\right) \leqq e^{-C \delta / \varepsilon_{n}}
$$

for any $\delta$ and $\varepsilon_{n}$ such that $\delta>2 \varepsilon_{n}$.

The proof is quite standard and we give it here just to keep the paper selfcontained. Let $r_{n}:=\left[\varepsilon_{n} n\right], R_{n}:=\left[\delta n / r_{n}\right]$, where $[x]$ is the integer part $x$. Clearly,

$$
R_{n} \geqq \frac{\delta}{\varepsilon_{n}}-1 .
$$

For $\mathbf{P}\left(\nu_{n}>\delta n\right)$ we have the following bound from above:

$$
\begin{aligned}
\mathbf{P}\left(\nu_{n}>\delta n\right) & \leqq \mathbf{P}\left(\left|S_{j r_{n}}\right|<c\left(\varepsilon_{n} n\right) \text { for all } j \leqq R_{n}\right) \\
& \leqq \mathbf{P}\left(\left|S_{j r_{n}}-S_{(j-1) n}\right|<2 c\left(\varepsilon_{n} n\right) \text { for all } j \leqq R_{n}\right) \\
& =\left(\mathbf{P}\left(\left|S_{r_{n}}\right|<2 c\left(\varepsilon_{n} n\right)\right)\right)^{R_{n}}
\end{aligned}
$$

Copyright $@$ ㅇ by SIAM. Unauthorized reproduction of this article is prohibited. 
Since $\left\{S_{n}\right\}$ is asymptotically stable, there exists a positive constant $C$ such that

$$
\mathbf{P}\left(\left|S_{r_{n}}\right|<2 c\left(\varepsilon_{n} n\right)\right) \leqq e^{-C}
$$

Combining this with (28) we get the proof of Lemma 2.

Proposition 3. Let $g$ be such that $g(0)>0$ and condition (12) holds. Then there exists a finite strictly positive limit

$$
V(g)=\lim _{n \rightarrow \infty} \mathbf{E}\left[h(S(n)+g(n)) ; T_{g}>n\right]<\infty .
$$
that

Proof. Since $h(S(n)+g(n)) 1\left\{T_{g}>n\right\}$ is a submartingale it is sufficient to show

$$
\sup _{n} \mathbf{E}\left[h(S(n)+g(n)) ; T_{g}>n\right]<\infty,
$$

and then apply the well-known submartingale convergence theorem.

Fix $n_{0}>1$ and let

$$
n_{m}=n_{0}\left[\left(\frac{1}{\delta}\right)^{m}\right], \quad m \geqq 1,
$$

where $[r]$ denotes the integer part of $r$. Since $h\left(S_{n}+g(n)\right) 1\left\{T_{g}>n\right\}$ is a positive submartingale, it suffices to show that the subsequence $\mathbf{E}\left[h\left(S_{n_{m}}+g\left(n_{m}\right)\right) ; T_{g}>n_{m}\right]$ is bounded. We first split the expectation into two parts,

$$
\begin{aligned}
\mathbf{E}\left[h\left(S_{n_{m}}+g\left(n_{m}\right)\right) ;\right. & \left.T_{g}>n_{m}\right] \\
=E_{1}+E_{2}:=\mathbf{E} & {\left[h\left(S_{n_{m}}+g\left(n_{m}\right)\right) ; T_{g}>n_{m}, \nu_{n_{m}} \leqq n_{m-1}\right] } \\
& +\mathbf{E}\left[h\left(S_{n_{m}}+g\left(n_{m}\right)\right) ; T_{g}>n_{m}, \nu_{n_{m}}>n_{m-1}\right] .
\end{aligned}
$$

For fixed $n$, let

$$
\widetilde{\tau}(g(n)):=\min \left\{k \geqq 1: S_{k}+g(n) \leqq 0\right\} .
$$

For the second expectation note that

$$
E_{2}=\mathbf{E}\left[\mathbf{E}\left[h\left(S_{n_{m}}+g\left(n_{m}\right)\right) 1\left\{T_{g}>n_{m}\right\} \mid \mathcal{F}_{n_{m-1}}\right] 1\left\{\nu_{n_{m}}>n_{m-1}\right\}\right] .
$$

Then, using the harmonicity of $h$, we obtain

$$
\begin{aligned}
\mathbf{E}[ & \left.h\left(S_{n_{m}}+g\left(n_{m}\right)\right) 1\left\{T_{g}>n_{m}\right\} \mid \mathcal{F}_{n_{m-1}}\right] \\
\leqq & \mathbf{E}_{S_{n_{m-1}}}\left[h\left(S_{n_{m}-n_{m-1}}+g\left(n_{m}\right)\right) 1\left\{\widetilde{\tau}\left(g\left(n_{m}\right)\right)>n_{m}-n_{m-1}\right\}\right] \\
& \times 1\left\{T_{g}>n_{m-1}\right\} \\
\leqq & h\left(S_{n_{m-1}}+g\left(n_{m}\right)\right) \leqq h\left(S_{n_{m-1}}+g\left(n_{m-1}\right)\right)+h\left(g\left(n_{m}\right)\right),
\end{aligned}
$$

where we used the subadditivity of the renewal function in the last step. Therefore,

$$
\begin{aligned}
E_{2} & \leqq \mathbf{E}\left[h\left(S_{n_{m-1}}\right)+g\left(n_{m-1}\right)+h\left(g\left(n_{m}\right)\right) ; \nu_{n_{m}}>n_{m-1}\right] \\
& \leqq \mathbf{E}\left[h\left(c\left(\varepsilon_{n_{m}} n_{m}\right)+g\left(n_{m}\right)\right)+h\left(g\left(n_{m}\right)\right) ; \nu_{n_{m}}>n_{m-1}\right] \\
& \leqq\left(h\left(c\left(\varepsilon_{n_{m}} n_{m}\right)\right)+2 h\left(g\left(n_{m}\right)\right)\right) \mathbf{P}\left(\nu_{n_{m}}>n_{m-1}\right) .
\end{aligned}
$$

Applying Lemma 10 with $\varepsilon_{n}:=(3 \delta C \log n)^{-1}$, we obtain

$$
E_{2} \leqq\left(h\left(c\left(\varepsilon_{n_{m}} n_{m}\right)\right)+2 h\left(g\left(n_{m}\right)\right)\right) n_{m}^{-3} .
$$

Copyright (c) by SIAM. Unauthorized reproduction of this article is prohibited. 
The first expectation can be estimated similarly. First, note

$$
E_{1}=\mathbf{E}\left[\mathbf{E}\left[h\left(S_{n_{m}}+g\left(n_{m}\right)\right) 1\left\{T_{g}>n_{m}\right\} \mid \mathcal{F}_{\nu_{n_{m}}}\right], \nu_{n_{m}} \leqq n_{m-1}\right] .
$$

Then, again, using the harmonicity of $h$, we obtain

$$
\begin{aligned}
\mathbf{E}[ & \left.h\left(S_{n_{m}}+g\left(n_{m}\right)\right) 1\left\{T_{g}>n_{m}\right\} \mid \mathcal{F}_{\nu_{n_{m}}}\right] \\
& \leqq \mathbf{E}_{S_{\nu_{m}}}\left[h\left(S_{n_{m}-\nu_{n_{m}}}+g\left(n_{m}\right)\right) 1\left\{\widetilde{\tau}\left(g\left(n_{m}\right)\right)>n_{m}-\nu_{n_{m}}\right\}\right] 1\left\{T_{g}>\nu_{n_{m}}\right\} \\
& \leqq h\left(S_{\nu_{n_{m}}}+g\left(n_{m}\right)\right) 1\left\{T_{g}>\nu_{n_{m}}\right\} \\
& \leqq\left(h\left(S_{\nu_{n_{m}}}+g\left(\nu_{n_{m}}\right)\right)+h\left(g\left(n_{m}\right)\right)\right) 1\left\{T_{g}>\nu_{n_{m}}\right\} .
\end{aligned}
$$

Since $S_{\nu_{n}} \geqq c\left(\varepsilon_{n} n\right)$, we have

$$
\begin{aligned}
& \mathbf{E}\left[h\left(S_{n_{m}}+g\left(n_{m}\right)\right) 1\left\{T_{g}>n_{m}\right\} \mid \mathcal{F}_{\nu_{n_{m}}}\right] \\
& \quad \leqq h\left(S_{\nu_{n_{m}}}+g\left(\nu_{n_{m}}\right)\right)\left(1+\frac{h\left(g\left(n_{m}\right)\right)}{h\left(c\left(\varepsilon_{n_{m}} n_{m}\right)\right)}\right) 1\left\{T_{g}>\nu_{n_{m}}\right\} .
\end{aligned}
$$

Hence, using the latter inequality and using the submartingale property, we obtain

$$
\begin{aligned}
E_{1} & \leqq\left(1+\frac{h\left(g\left(n_{m}\right)\right)}{h\left(c\left(\varepsilon_{n_{m}} n_{m}\right)\right)}\right) \mathbf{E}\left[h\left(S_{\nu_{n_{m}}}+g\left(\nu_{n_{m}}\right)\right) ; T_{g}>\nu_{n_{m}}, \nu_{n_{m}} \leqq n_{m-1}\right] \\
& \leqq\left(1+\frac{h\left(g\left(n_{m}\right)\right)}{h\left(c\left(\varepsilon_{n_{m}} n_{m}\right)\right)}\right) \mathbf{E}\left[h\left(S_{n_{m-1}}+g\left(n_{m-1}\right)\right) ; T_{g}>n_{m-1}\right] .
\end{aligned}
$$

As a result we arrive at the following estimate:

$$
\begin{aligned}
\mathbf{E}[ & \left.h\left(S_{n_{m}}+g\left(n_{m}\right)\right) ; T_{g}>n_{m}\right] \\
\leqq & \left(1+\frac{h\left(g\left(n_{m}\right)\right)}{h\left(c\left(\varepsilon_{n_{m}} n_{m}\right)\right)}\right) \mathbf{E}\left[h\left(S_{n_{m-1}}+g\left(n_{m-1}\right)\right) ; T_{g}>n_{m-1}\right] \\
& +\frac{h\left(c\left(\varepsilon_{n_{m}} n_{m}\right)\right)+2 h\left(g\left(n_{m}\right)\right)}{n_{m}^{3}} .
\end{aligned}
$$

Iterating this procedure $m$ times we obtain

$$
\begin{aligned}
\mathbf{E}\left[h\left(S_{n_{m}}+g\left(n_{m}\right)\right) ; T_{g}>n_{m}\right] \leqq \prod_{j=1}^{m}\left(1+\frac{h\left(g\left(n_{j}\right)\right)}{h\left(c\left(\varepsilon_{n_{j}} n_{j}\right)\right)}\right) \\
\quad \times\left(\mathbf{E}\left[h\left(S_{n_{0}}+g\left(n_{0}\right)\right) ; T_{g}>n_{0}\right]+\sum_{j=1}^{m} \frac{h\left(c\left(\varepsilon_{n_{j}} n_{j}\right)\right)+2 h\left(g\left(n_{j}\right)\right)}{n_{j}^{3}}\right) .
\end{aligned}
$$

Now note that (20) implies

$$
\sum_{j=1}^{\infty} \frac{h\left(g\left(n_{j}\right)\right)}{h\left(c\left(\varepsilon_{n_{j}} n_{j}\right)\right)}<\infty .
$$

Therefore, the product

$$
\prod_{j=1}^{\infty}\left(1+\frac{h\left(g\left(n_{j}\right)\right)}{h\left(c\left(\varepsilon_{n_{j}} n_{j}\right)\right)}\right)
$$

Copyright (c) by SIAM. Unauthorized reproduction of this article is prohibited. 
is finite. Furthermore, recalling that the index of $h$ is $\alpha(1-\rho)$, we conclude that

$$
\sum_{j=0}^{\infty} \frac{h\left(c\left(\varepsilon_{n_{j}} n_{j}\right)\right)+2 h\left(g\left(n_{j}\right)\right)}{n_{j}^{3}}<\infty .
$$

Then the statement of Proposition 3 immediately follows.

3.2. Derivation of the asymptotics. In the proof of (11) we will require the following result.

Lemma 3. Let $X \in \mathcal{D}(\alpha, \rho)$. Then,

$$
\mathbf{P}\left(T_{x}>n\right) \sim h(x) n^{\rho-1} L(n)
$$

uniformly in $x$ such that $x / c(n) \rightarrow 0$. Moreover, the following estimate holds for all $x \geqq 0$ :

$$
\mathbf{P}\left(T_{x}>n\right) \leqq C h(x) n^{\rho-1} L(n) .
$$

Proof. Statement (29) is Corollary 3 in [5].

Let

$$
\sigma(x):=\min \left\{k \geqq 1: \sum_{i=1}^{k} \chi_{i}>x\right\}
$$

Then

$$
\begin{aligned}
\mathbf{P}\left(T_{x}>n\right) & \leqq \mathbf{P}\left(\sum_{k=1}^{\sigma(x)} \tau_{k}>n\right) \leqq \mathbf{P}\left(\sum_{k=1}^{\sigma(x)}\left(\tau_{k} \wedge n\right)>n\right) \\
& \leqq \frac{1}{n} \mathbf{E} \sum_{k=1}^{\sigma(x)}\left(\tau_{k} \wedge n\right)=\frac{1}{n} \mathbf{E} \sigma(x) \mathbf{E}\left(T_{0} \wedge n\right),
\end{aligned}
$$

where we applied the Wald identity in the last step. Since the tail of $T_{0}$ is regularly varying of index $\rho-1 \in(0,1)$, by the Tauberian theorem, $\mathbf{E}\left(T_{0} \wedge n\right) \sim \rho^{-1} n \mathbf{P}(\tau>n)$. Also note that $h(x)=\mathbf{E} \sigma(x)$. Hence, as $n \rightarrow \infty$

$$
\mathbf{P}\left(T_{x}>n\right) \leqq(1+o(1)) h(x) \rho^{-1} \mathbf{P}\left(T_{0}>n\right)
$$

uniformly in $x$.

According to (20),

$$
\int_{x}^{2 x} \frac{h(g(y))}{y h(c(y / \log y))} d y \rightarrow 0 \quad \text { as } x \rightarrow \infty .
$$

Since $c(x), h(x), g(x)$ increase, and, in addition, $h(x)$ and $c(x)$ are regularly varying, we conclude that

$$
\frac{h(g(x))}{h(c(x / \log x))} \rightarrow 0 .
$$

In other words, $g(x)=o(c(x / \log x))$. Consequently, there exists a sequence $\delta_{n} \rightarrow 0$ such that $g(n)=o\left(c\left(\delta_{n} n / \log n\right)\right)$. Moreover, we may assume that $\delta_{n}$ is such that for 
slowly varying functions $L$ and $l$ from $\mathbf{P}(\tau>n) \sim n^{\rho-1} L(n)$ and $h(x)=x^{\alpha(1-\rho)} l(x)$ the following asymptotics equivalences hold: $l\left(\delta_{n} n\right) \sim l(n)$ and $L\left(\delta_{n} n\right) \sim L(n)$.

Applying Lemma 2 with $\delta=\delta_{n}$ and $\varepsilon_{n}=C \delta_{n} /(2 \log n)$, we obtain

$$
\mathbf{P}\left(T_{g}>n\right)=\mathbf{P}\left(T_{g}>n, \nu_{n} \leqq n \delta_{n}\right)+O\left(n^{-2}\right) .
$$

This immediately gives us the upper bound. Indeed,

$$
\begin{aligned}
\mathbf{P} & \left(T_{g}>n, \nu_{n} \leqq n \delta_{n}\right) \\
\leqq & \int_{c_{n \delta_{n}}}^{\infty} \mathbf{P}\left(T_{g}>\nu_{n}, S_{\nu_{n}} \in d y, \nu_{n} \leqq n \delta_{n}\right) \mathbf{P}\left(\widetilde{\tau}(y+g(n))>n-n \delta_{n}\right) \\
\leqq & C \int_{c_{n \delta_{n}}}^{\infty} \mathbf{P}\left(T_{g}>\nu_{n}, S_{\nu_{n}} \in d y, \nu_{n} \leqq n \delta_{n}\right) \\
& \times h(y+g(n))\left(n-n \delta_{n}\right)^{\rho-1} L\left(n-n \delta_{n}\right),
\end{aligned}
$$

where we applied (30) to derive the last inequality. Now note that

$$
h(y+g(n)) \sim h(y) \quad\left(n-n \delta_{n}\right)^{\rho-1} L\left(n-n \delta_{n}\right) \sim n^{\rho-1} L(n)
$$

uniformly in $y>c\left(\delta_{n} n / \log n\right)$. Therefore, for large $n$ we have

$$
\begin{aligned}
\mathbf{P}\left(T_{g}>n, \nu_{n} \leqq n \delta_{n}\right) \leqq & C \int_{\varepsilon c_{n \delta_{n}}}^{\infty} \mathbf{P}\left(T_{g}>\nu_{n}, S_{\nu_{n}} \in d y, \nu_{n} \leqq n \delta_{n}\right) \\
& \times h(y) \mathbf{P}\left(T_{0}>n\right) \\
\leqq & C \mathbf{E}\left[h\left(S_{\nu_{n}}\right) ; T_{g}>\nu_{n}, \nu_{n} \leqq n \delta_{n}\right] \mathbf{P}\left(T_{0}>n\right) .
\end{aligned}
$$

By the submartingale property and Proposition 3,

$$
\mathbf{E}\left[h\left(S_{\nu_{n}}\right) ; T_{g}>\nu_{n}, \nu_{n} \leqq n \delta_{n}\right] \leqq \mathbf{E}\left[h\left(S_{n \delta_{n}}\right) ; T_{g}>n \delta_{n}\right] \leqq V(g) .
$$

Consequently,

$$
\mathbf{P}\left(T_{g}>n, \nu_{n} \leqq n \delta_{n}\right) \leqq C V(g) \mathbf{P}\left(T_{0}>n\right) .
$$

Combining this with (31), we obtain

$$
\mathbf{P}\left(T_{g}>n\right) \leqq C V(g) \mathbf{P}\left(T_{0}>n\right) .
$$

Further, we are going to improve the bound (33) to obtain sharp asymptotics. Let the sequence $A_{n} \uparrow \infty$ be such that $A_{n} c\left(\delta_{n} n / \log n\right)=o(c(n))$. Set, for brevity, $r_{n}:=c\left(\delta_{n} n / \log n\right)$. We split the probability $\mathbf{P}\left(T_{g}>n, \nu_{n} \leqq \delta_{n} n\right)$ into two parts

$$
\begin{aligned}
\mathbf{P}\left(T_{g}>n, \nu_{n} \leqq \delta_{n} n\right)= & \mathbf{P}\left(T_{g}>n, \nu_{n} \leqq \delta_{n} n, S_{\nu_{n}} \in\left(r_{n}, A_{n} r_{n}\right)\right) \\
& +\mathbf{P}\left(T_{g}>n, \nu_{n} \leqq \delta_{n} n, S_{\nu_{n}}>A_{n} r_{n}\right) .
\end{aligned}
$$

We apply (29) to the first summand. We have

$$
\begin{aligned}
\mathbf{P}\left(T_{g}\right. & \left.>n, \nu_{n} \leqq \delta_{n} n, S_{\nu_{n}} \in\left(r_{n}, A_{n} r_{n}\right)\right) \\
& \leqq \int_{r_{n}}^{A_{n} r_{n}} \mathbf{P}\left(T_{g}>\nu_{n}, S_{\nu_{n}} \in d y, \nu_{n} \leqq n \delta_{n}\right) \mathbf{P}\left(\widetilde{\tau}_{y+g(n)}>n-n \delta_{n}\right) \\
& \sim \int_{r_{n}}^{A_{n} r_{n}} \mathbf{P}\left(T_{g}>\nu_{n}, S_{\nu_{n}} \in d y, \nu_{n} \leqq n \delta_{n}\right) h(y) \mathbf{P}\left(T_{0}>n\right) \\
& \leqq \mathbf{E}\left[h\left(S_{\nu_{n}}\right) ; T_{g}>\nu_{n}, \nu_{n} \leqq n \delta_{n}\right] \mathbf{P}\left(T_{0}>n\right) \\
& \leqq V(g) \mathbf{P}\left(T_{0}>n\right),
\end{aligned}
$$

Copyright $@$ by SIAM. Unauthorized reproduction of this article is prohibited. 
where we used (32) in the last step. The bound from below can be obtained by a similar argument,

$$
\begin{aligned}
\mathbf{P}\left(T_{g}\right. & \left.>n, \nu_{n} \leqq \delta_{n} n, S_{\nu_{n}} \in\left(r_{n}, A_{n} r_{n}\right)\right) \\
& \geqq \int_{r_{n}}^{A_{n} r_{n}} \mathbf{P}\left(T_{g}>\nu_{n}, S_{\nu_{n}} \in d y, \nu_{n} \leqq n \delta_{n}\right) \mathbf{P}\left(\widetilde{\tau}_{y}>n-n \delta_{n}\right) \\
& \sim \int_{r_{n}}^{A_{n} r_{n}} \mathbf{P}\left(T_{g}>\nu_{n}, S_{\nu_{n}} \in d y, \nu_{n} \leqq n \delta_{n}\right) h(y) \mathbf{P}\left(T_{0}>n\right) \\
& =\mathbf{E}\left[h\left(S_{\nu_{n}}\right) ; T_{g}>\nu_{n}, S_{\nu_{n}}<A_{n} r_{n}, \nu_{n} \leqq n \delta_{n}\right] \mathbf{P}\left(T_{0}>n\right) .
\end{aligned}
$$

Now we turn to the second summand in (34). Using the Markov property and (29), we obtain

$$
\begin{aligned}
& \mathbf{P}\left(T_{g}>n, \nu_{n} \leqq \delta_{n} n, S_{\nu_{n}}>A_{n} r_{n}, T_{g}>\nu_{n}\right) \\
& \quad \leqq C \mathbf{E}\left[h\left(S_{\nu_{n}}\right) ; S_{\nu_{n}}>A_{n} r_{n}, \nu_{n} \leqq \delta_{n} n, T_{g}>\nu_{n}\right] \mathbf{P}\left(T_{0}>n\right) .
\end{aligned}
$$

So it is left to prove that

$$
\mathbf{E}\left[h\left(S_{\nu_{n}}\right) ; S_{\nu_{n}}>A_{n} r_{n}, \nu_{n} \leqq \delta_{n} n, T_{g}>\nu_{n}\right] \rightarrow 0 .
$$

Now note that $S_{\nu_{n}-1} \leqq r_{n}$. Then on the event $\left\{S_{\nu_{n}}>A_{n} r_{n}\right\}$ we have

$$
X_{\nu_{n}}>\left(A_{n}-1\right) r_{n}>\frac{A_{n}}{2} r_{n}>S_{\nu_{n}-1} .
$$

Hence $S_{\nu_{n}}=X_{\nu_{n}}+S_{\nu_{n}-1} \leqq 2 X_{\nu_{n}}$. Using the subadditivity and monotonic increase of $h$ we obtain

$$
h\left(S_{\nu_{n}}\right) \leqq h\left(2 X_{\nu_{n}}\right) \leqq 2 h\left(X_{\nu_{n}}\right)
$$

Therefore,

$$
\begin{aligned}
E & :=\mathbf{E}\left[h\left(S_{\nu_{n}}\right) ; S_{\nu_{n}}>A_{n} r_{n}, \nu_{n} \leqq \delta_{n} n, T_{g}>\nu_{n}\right] \\
& \leqq 2 \mathbf{E}\left[h\left(X_{\nu_{n}}\right) ; X_{\nu_{n}}>\frac{A_{n}}{2} r_{n}, \nu_{n} \leqq \delta_{n} n, T_{g}>\nu_{n}\right] \\
& \leqq \sum_{k=1}^{\delta_{n} n} \mathbf{E}\left[h\left(X_{k}\right) ; X_{k}>\frac{A_{n}}{2} r_{n}, \nu_{n}=k, T_{g}>k\right] \\
& \leqq 2 \sum_{k=1}^{\delta_{n} n} \mathbf{P}\left(T_{g} \geqq k\right) \mathbf{E}\left[h(X) ; X>\frac{A_{n}}{2} c_{n \delta_{n}}\right] .
\end{aligned}
$$

Applying (33), we obtain

$$
\begin{aligned}
E & \leqq C \sum_{k=1}^{\delta_{n} n} \mathbf{P}\left(T_{0} \geqq k\right) \mathbf{E}\left[h(X) ; X>\frac{A_{n}}{2} r_{n}\right] \\
& \leqq C \delta_{n} n \mathbf{P}\left(T_{0}>\delta_{n} n\right) \mathbf{E}\left[h(X) ; X>\frac{A_{n}}{2} r_{n}\right] .
\end{aligned}
$$

Recall that if $X \in \mathcal{D}(\alpha, \beta)$, then the distribution function of $\theta(d u):=u^{2} \mathbf{P}(|X| \in d u)$ is regularly varying of index $2-\alpha$, that is,

$$
\Theta(x):=\theta((0, x))=x^{2-\alpha} \ell(x)
$$

Copyright (c) by SIAM. Unauthorized reproduction of this article is prohibited. 
Therefore,

$$
\mathbf{E}\left[h(X) ; X>\frac{A_{n}}{2} r_{n}\right] \leqq \int_{\left(A_{n} / 2\right) r_{n}}^{\infty} \frac{h(x)}{x^{2}} \theta(d x) \leqq C \frac{h\left(A_{n} r_{n}\right)}{\left(A_{n} r_{n}\right)^{2}} \Theta\left(A_{n} r_{n}\right) .
$$

We can pick a sequence $A_{n}$ such that

$$
\frac{h\left(A_{n} r_{n}\right)}{\left(A_{n} r_{n}\right)^{2}} \Theta\left(A_{n} r_{n}\right) \sim\left(\frac{c(n)}{A_{n} r_{n}}\right)^{\alpha \rho} \frac{h(c(n))}{c^{2}(n)} \Theta(c(n)) .
$$

Then

$$
E \leqq C \delta_{n}^{\rho} n \mathbf{P}\left(T_{0}>n\right)\left(\frac{c(n)}{A_{n} r_{n}}\right)^{\alpha \rho} \frac{h(c(n))}{c^{2}(n)} \Theta(c(n)) .
$$

Now recall that

$$
h\left(c_{n}\right) \mathbf{P}\left(T_{0}>n\right) \rightarrow C_{0}, \quad n \frac{\Theta(c(n))}{c^{2}(n)} \rightarrow 1
$$

for some constant $C_{0}$. Hence, for an appropriate choice of $A_{n}$,

$$
E \leqq C \delta_{n}^{\rho}\left(\frac{c(n)}{A_{n} r_{n}}\right)^{\alpha \rho} \rightarrow 0, \quad n \rightarrow \infty .
$$

This completes the proof of (37).

Noting that (37) yields

$$
\mathbf{P}\left(T_{g}>n, \nu_{n} \leqq \delta_{n} n, S_{\nu_{n}}>A_{n} r_{n}, T_{g}>\nu_{n}\right)=o\left(\mathbf{P}\left(T_{0}>n\right)\right)
$$

and taking into account (35), we obtain

$$
\limsup _{n \rightarrow \infty} \frac{\mathbf{P}\left(T_{g}>n\right)}{\mathbf{P}\left(T_{0}>n\right)} \leqq V(g) .
$$

Combining (36) and (37), we have

$$
\liminf _{n \rightarrow \infty} \frac{\mathbf{P}\left(T_{g}>n\right)}{\mathbf{P}\left(T_{0}>n\right)} \geqq \liminf _{n \rightarrow \infty} \mathbf{E}\left[h\left(S_{\nu_{n}}\right) ; T_{g}>\nu_{n}, \nu_{n} \leqq n \delta_{n}\right] .
$$

It follows from Lemma 2 with $\delta=\delta_{n}$ and $\varepsilon_{n}=C \delta_{n} /(2 \log n)$ that

$$
\mathbf{E}\left[h\left(S_{\delta_{n} n}+g\left(\delta_{n} n\right)\right) ; T_{g}>\delta_{n} n, \nu_{n}>n \delta_{n}\right] \leqq h(c(n)) \mathbf{P}\left(\nu_{n}>n \delta_{n}\right) \rightarrow 0 .
$$

Consequently,

$$
\mathbf{E}\left[h\left(S_{\nu_{n}}\right) ; T_{g}>\nu_{n}, \nu_{n} \leqq n \delta_{n}\right]=\mathbf{E}\left[h\left(S_{\theta_{n}}+g\left(\theta_{n}\right)\right) ; T_{g}>\theta_{n}\right]+o(1),
$$

where $\theta_{n}:=\nu_{n} \wedge \delta_{n} n$. Applying the optional stopping theorem to the submartingale $h\left(S_{n}+g(n)\right) 1\left\{T_{g}>n\right\}$, we obtain for each fixed $N$

$$
\mathbf{E}\left[h\left(S_{\theta_{n}}+g\left(\theta_{n}\right)\right) ; T_{g}>\theta_{n}\right] \geqq \mathbf{E}\left[h\left(S_{\theta_{n} \wedge N}+g\left(\theta_{n} \wedge N\right)\right) ; T_{g}>\theta_{n} \wedge N\right] .
$$


Note also that

$$
\begin{aligned}
& \mid \mathbf{E}\left[h\left(S_{\theta_{n} \wedge N}+g\left(\theta_{n} \wedge N\right)\right) ; T_{g}>\theta_{n} \wedge N, \nu_{n}<N\right] \\
&-\mathbf{E}\left[h\left(S_{N}+g(N)\right) ; T_{g}>N, \nu_{n}<N\right] \mid \\
& \leqq \mathbf{E}\left[h\left(\max _{k<N}\left|S_{k}\right|+g(N)\right) ; \max _{k<N}\left|S_{k}\right|>c\left(\frac{\delta_{n} n}{\log n}\right)\right] \rightarrow 0 .
\end{aligned}
$$

As a result we have

$$
\mathbf{E}\left[h\left(S_{\theta_{n}}+g\left(\theta_{n}\right)\right) ; T_{g}>\theta_{n}\right] \geqq \mathbf{E}\left[h\left(S_{N}+g(N)\right) ; T_{g}>N\right]+o(1)
$$

and, consequently,

$$
\liminf _{n \rightarrow \infty} \mathbf{E}\left[h\left(S_{\nu_{n}}\right) ; T_{g}>\nu_{n}, \nu_{n} \leqq n \delta_{n}\right] \geqq \mathbf{E}\left[h\left(S_{N}+g(N)\right) ; T_{g}>N\right] .
$$

Letting $N$ to infinity we obtain

$$
\liminf _{n \rightarrow \infty} \mathbf{E}\left[h\left(S_{\nu_{n}}\right) ; T_{g}>\nu_{n}, \nu_{n} \leqq n \delta_{n}\right] \geqq V(g) .
$$

Combining this with (38), we complete the proof of Theorem 2.

4. Proof of Theorem 3. The proof follows closely the proof of Theorem 2.

\subsection{Positivity of $\widehat{V}(g)$.}

Lemma 4. The sequence $Y_{n}=h\left(S_{n}-g(n)\right) 1\left\{\widehat{T}_{g}>n\right\}$ is a supermartingale.

Proof. Clearly,

$$
\begin{aligned}
\mathbf{E}\left[Y_{n+1}-Y_{n} \mid \mathcal{F}_{n}\right]=\mathbf{E}[ & \left(h\left(S_{n+1}-g(n+1)\right)-h\left(S_{n}-g(n)\right)\right) \\
& \left.\times 1\left\{\widehat{T}_{g}>n\right\} \mid \mathcal{F}_{n}\right] \\
-\mathbf{E}[ & \left.h\left(S_{n+1}-g(n+1)\right) 1\left\{\widehat{T}_{g}=n+1\right\} \mid \mathcal{F}_{n}\right] .
\end{aligned}
$$

Note that $h\left(S_{n+1}-g(n+1)\right) 1\left\{\widehat{T}_{g}=n+1\right\}=0$, since $h(x)=0$ for $x \leqq 0$. Further, by harmonicity of $h$,

$$
\begin{aligned}
& \mathbf{E}\left[Y_{n+1}-Y_{n} \mid \mathcal{F}_{n}\right] \\
& \quad=\mathbf{E}\left[\left(h\left(S_{n+1}-g(n+1)\right)\right) 1\left\{\widehat{T}_{g}>n\right\} \mid \mathcal{F}_{n}\right]-h\left(S_{n}-g(n)\right) 1\left\{\widehat{T}_{g}>n\right\} \\
& \quad=h\left(S_{n}-g(n+1)\right) 1\left\{\widehat{T}_{g}>n\right\}-h\left(S_{n}-g(n)\right) 1\left\{\widehat{T}_{g}>n\right\} \leqq 0,
\end{aligned}
$$

since $h$ and $g$ are monotonically increasing. Lemma 4 is proved.

By the supermartingale property, the $\operatorname{limit}_{n \rightarrow \infty} \mathbf{E}\left[h\left(S_{n}-g(n)\right) ; \widehat{T}_{g}>n\right]$ exists and is finite. Thus, it is sufficient to show that this limit is positive.

Proposition 4. Assume that $g$ is such that $g(0)>0$ and condition (12) holds. Then there exists a strictly positive and finite limit

$$
\widehat{V}(g):=\lim _{n \rightarrow \infty} \mathbf{E}\left[h(S(n)-g(n)) ; \widehat{T}_{g}>n\right] .
$$

Proof. Since $h(S(n)-g(n)) 1\left\{\widehat{T}_{g}>n\right\}$ is a supermartingale it is sufficient to show that

$$
\inf _{n} \mathbf{E}\left[h(S(n)-g(n)) ; \widehat{T}_{g}>n\right]>0
$$

Copyright ( $\odot$ by SIAM. Unauthorized reproduction of this article is prohibited. 
Fix $n_{0}>1$ and let

$$
n_{m}=n_{0}\left[\left(\frac{1}{\delta}\right)^{m}\right], \quad m \geqq 1
$$

where $[r]$ denotes the integer part of $r$. Since $\mathbf{E}\left[h\left(S_{n}-g(n)\right) ; \widehat{T}_{g}>n\right]$ is decreasing, it suffices to show that $\inf _{m} \mathbf{E}\left[h\left(S_{n_{m}}-g\left(n_{m}\right)\right) ; \widehat{T}_{g}>n_{m}\right]>0$. We have

$$
\begin{aligned}
\mathbf{E}\left[h\left(S_{n_{m}}-g\left(n_{m}\right)\right) ; \widehat{T}_{g}>n_{m}\right] \geqq E_{1} \\
\quad:=\mathbf{E}\left[h\left(S_{n_{m}}-g\left(n_{m}\right)\right) ; \widehat{T}_{g}>n_{m}, \nu_{n_{m}} \leqq n_{m-1}\right] .
\end{aligned}
$$

For fixed $n$, let

$$
\widetilde{\tau}(g(n)):=\min \left\{k \geqq 1: S_{k}-g(n) \leqq 0\right\} .
$$

Then

$$
E_{1}=\mathbf{E}\left[\mathbf{E}\left[h\left(S_{n_{m}}-g\left(n_{m}\right)\right) 1\left\{\widehat{T}_{g}>n_{m}\right\} \mid \mathcal{F}_{\nu_{n_{m}}}\right], \nu_{n_{m}} \leqq n_{m-1}\right] .
$$

Using the harmonicity of $h$ we obtain

$$
\begin{aligned}
\mathbf{E}[ & \left.h\left(S_{n_{m}}-g\left(n_{m}\right)\right) 1\left\{\widehat{T}_{g}>n_{m}\right\} \mid \mathcal{F}_{\nu_{n_{m}}}\right] \\
& \geqq \mathbf{E}_{S_{\nu_{n_{m}}}}\left[h\left(S_{n_{m}-\nu_{n_{m}}}-g\left(n_{m}\right)\right) 1\left\{\widetilde{\tau}\left(g\left(n_{m}\right)\right)>n_{m}-\nu_{n_{m}}\right\}\right] 1\left\{\widehat{T}_{g}>\nu_{n_{m}}\right\} \\
\quad & \geqq h\left(S_{\nu_{n_{m}}}-g\left(n_{m}\right)\right) 1\left\{\widehat{T}_{g}>\nu_{n_{m}}\right\} \\
\quad & \geqq\left(h\left(S_{\nu_{n_{m}}}-g\left(\nu_{n_{m}}\right)\right)-h\left(g\left(n_{m}\right)\right)\right) 1\left\{\widehat{T}_{g}>\nu_{n_{m}}\right\}
\end{aligned}
$$

where we used the subadditivity of $h$ in the last inequality. Now, since $S_{\nu_{n}} \geqq c\left(\varepsilon_{n} n\right)$, we have

$$
\begin{aligned}
& \mathbf{E}\left[h\left(S_{n_{m}}-g\left(n_{m}\right)\right) 1\left\{\widehat{T}_{g}>n_{m}\right\} \mid \mathcal{F}_{\nu_{n_{m}}}\right] \\
& \quad \geqq h\left(S_{\nu_{n_{m}}}-g\left(\nu_{n_{m}}\right)\right)\left(1-\frac{h\left(g\left(n_{m}\right)\right)}{h\left(c\left(\varepsilon_{n_{m}} n_{m}\right)\right)}\right) 1\left\{\widehat{T}_{g}>\nu_{n_{m}}\right\} .
\end{aligned}
$$

Hence, using the latter inequality and the submartingale property, we have

$$
\begin{aligned}
E_{1} \geqq & \left(1-\frac{h\left(g\left(n_{m}\right)\right)}{h\left(c\left(\varepsilon_{n_{m}} n_{m}\right)\right)}\right) \mathbf{E}\left[h\left(S_{\nu_{n_{m}}}-g\left(\nu_{n_{m}}\right)\right) ; \widehat{T}_{g}>\nu_{n_{m}}, \nu_{n_{m}} \leqq n_{m-1}\right] \\
\geqq & \left(1-\frac{h\left(g\left(n_{m}\right)\right)}{h\left(c\left(\varepsilon_{n_{m}} n_{m}\right)\right)}\right) \mathbf{E}\left[h\left(S_{n_{m-1}}-g\left(n_{m-1}\right)\right) ; \widehat{T}_{g}>n_{m-1}, \nu_{n_{m}} \leqq n_{m-1}\right] \\
\geqq & \left(1-\frac{h\left(g\left(n_{m}\right)\right)}{h\left(c\left(\varepsilon_{n_{m}} n_{m}\right)\right)}\right) \mathbf{E}\left[h\left(S_{n_{m-1}}-g\left(n_{m-1}\right)\right) ; \widehat{T}_{g}>n_{m-1}\right] \\
& \quad-\mathbf{E}\left[h\left(S_{n_{m-1}}-g\left(n_{m-1}\right)\right) ; \widehat{T}_{g}>n_{m-1}, \nu_{n_{m}}>n_{m-1}\right] .
\end{aligned}
$$

Now note that

$$
\begin{aligned}
E_{2} & :=\mathbf{E}\left[h\left(S_{n_{m-1}}-g\left(n_{m-1}\right)\right) ; \widehat{T}_{g}>n_{m-1}, \nu_{n_{m}}>n_{m-1}\right] \\
& \leqq \mathbf{E}\left[h\left(c\left(\varepsilon_{n_{m}} n_{m}\right)\right) ; \nu_{n_{m}}>n_{m-1}\right] \\
& \leqq h\left(c\left(\varepsilon_{n_{m}} n_{m}\right)\right) \mathbf{P}\left(\nu_{n_{m}}>n_{m-1}\right) .
\end{aligned}
$$

Copyright $@$ by SIAM. Unauthorized reproduction of this article is prohibited. 
Applying Lemma 10 with $\varepsilon_{n}:=(3 \delta C \log n)^{-1}$, we obtain

$$
E_{2} \leqq h\left(c\left(\varepsilon_{n_{m}} n_{m}\right)\right) n_{m}^{-3} .
$$

As a result we have

$$
\begin{aligned}
& \mathbf{E}\left[h\left(S_{n_{m}}-g\left(n_{m}\right)\right) ; \widehat{T}_{g}>n_{m}\right] \\
& \geqq\left(1-\frac{h\left(g\left(n_{m}\right)\right)}{h\left(c\left(\varepsilon_{n_{m}} n_{m}\right)\right)}\right) \mathbf{E}\left[h\left(S_{n_{m-1}}-g\left(n_{m-1}\right)\right) ; \widehat{T}_{g}>n_{m-1}\right] \\
& \quad-\frac{h\left(c\left(\varepsilon_{n_{m}} n_{m}\right)\right)}{n_{m}^{3}} .
\end{aligned}
$$

Iterating this procedure $m$ times we obtain

$$
\begin{array}{r}
\mathbf{E}\left[h\left(S_{n_{m}}-g\left(n_{m}\right)\right) ; \widehat{T}_{g}>n_{m}\right] \geqq \prod_{j=1}^{m}\left(1-\frac{h\left(g\left(n_{j}\right)\right)}{h\left(c\left(\varepsilon_{n_{j}} n_{j}\right)\right)}\right) \\
\times\left(\mathbf{E}\left[h\left(S_{n_{0}}+g\left(n_{0}\right)\right) ; \widehat{T}_{g}>n_{0}\right]-\sum_{j=1}^{m} \frac{h\left(c\left(\varepsilon_{n_{j}} n_{j}\right)\right)}{n_{j}^{3}}\right) .
\end{array}
$$

Now note that (20) implies

$$
\sum_{j=1}^{\infty} \frac{h\left(g\left(n_{j}\right)\right)}{h\left(c\left(\varepsilon_{n_{j}} n_{j}\right)\right)}<\infty .
$$

Therefore, the product

$$
\prod_{j=1}^{\infty}\left(1-\frac{h\left(g\left(n_{j}\right)\right)}{h\left(c\left(\varepsilon_{n_{j}} n_{j}\right)\right)}\right)
$$

is finite. Furthermore, recalling that the index of regular variation of $h$ is equal to $\alpha(1-\rho)$, we conclude that

$$
\sum_{j=0}^{\infty} \frac{h\left(c\left(\varepsilon_{n_{j}} n_{j}\right)\right)}{n_{j}^{3}}<\infty .
$$

Proposition 4 is proved.

4.2. Derivation of the asymptotics. For $\widehat{T}_{g}$ the upper bound is obvious,

$$
\mathbf{P}\left(\widehat{T}_{g}>n\right) \leqq \mathbf{P}\left(T_{0}>n\right) .
$$

Let $\delta_{n}$ and $A_{n}$ be as in the proof of Theorem 2. Similarly to (31) and (34) we have

$$
\begin{aligned}
\mathbf{P}\left(\widehat{T}_{g}>n\right)= & \mathbf{P}\left(\widehat{T}_{g}>n, \nu_{n} \leqq n \delta_{n}\right)+O\left(n^{-2}\right) \\
\mathbf{P}\left(\widehat{T}_{g}>n, \nu_{n} \leqq \delta_{n} n\right)= & \mathbf{P}\left(\widehat{T}_{g}>n, \nu_{n} \leqq \delta_{n} n, S_{\nu_{n}} \in\left(r_{n}, A_{n} r_{n}\right)\right) \\
& +\mathbf{P}\left(\widehat{T}_{g}>n, \nu_{n} \leqq \delta_{n} n, S_{\nu_{n}}>A_{n} r_{n}\right) .
\end{aligned}
$$

Copyright $@$ ㅇ by SIAM. Unauthorized reproduction of this article is prohibited. 
We apply (29) to the first summand. We have

$$
\begin{aligned}
& \mathbf{P}\left(\widehat{T}_{g}>n, \nu_{n} \leqq \delta_{n} n, S_{\nu_{n}} \in\left(r_{n}, A_{n} r_{n}\right)\right) \\
& \quad \leqq \int_{r_{n}}^{A_{n} r_{n}} \mathbf{P}\left(\widehat{T}_{g}>\nu_{n}, S_{\nu_{n}} \in d y, \nu_{n} \leqq n \delta_{n}\right) \mathbf{P}\left(\widetilde{\tau}_{y}>n-n \delta_{n}\right) \\
& \quad \sim \int_{r_{n}}^{A_{n} r_{n}} \mathbf{P}\left(\widehat{T}_{g}>\nu_{n}, S_{\nu_{n}} \in d y, \nu_{n} \leqq n \delta_{n}\right) h(y) \mathbf{P}\left\{T_{0}>n\right\} \\
& \leqq \mathbf{E}\left[h\left(S_{\nu_{n}}\right) ; \widehat{T}_{g}>\nu_{n}\right] \mathbf{P}\left(T_{0}>n\right) \\
& \sim \widehat{V}(g) \mathbf{P}\left(T_{0}>n\right) .
\end{aligned}
$$

The bound from below can be obtained by a similar argument,

$$
\begin{aligned}
\mathbf{P} & \left(\widehat{T}_{g}>n, \nu_{n} \leqq \delta_{n} n, S_{\nu_{n}} \in\left(r_{n}, A_{n} r_{n}\right)\right) \\
& \geqq \int_{r_{n}}^{A_{n} r_{n}} \mathbf{P}\left(\widehat{T}_{g}>\nu_{n}, S_{\nu_{n}} \in d y, \nu_{n} \leqq n \delta_{n}\right) \mathbf{P}\left(\widetilde{\tau}_{y-g(n)}>n-n \delta_{n}\right) \\
& \sim \int_{r_{n}}^{A_{n} r_{n}} \mathbf{P}\left(\widehat{T}_{g}>\nu_{n}, S_{\nu_{n}} \in d y, \nu_{n} \leqq n \delta_{n}\right) h(y) \mathbf{P}\left(T_{0}>n\right) \\
& =\mathbf{E}\left[h\left(S_{\nu_{n}}\right) ; \widehat{T}_{g}>\nu_{n}, S_{\nu_{n}}<A_{n} r_{n}, \nu_{n} \leqq n \delta_{n}\right] \mathbf{P}\left(T_{0}>n\right) .
\end{aligned}
$$

Now we turn to the second summand in (42). Using the Markov property and (29), we obtain

$$
\begin{aligned}
& \mathbf{P}\left(\widehat{T}_{g}>n, \nu_{n} \leqq \delta_{n} n, S_{\nu_{n}}>A_{n} r_{n}, \widehat{T}_{g}>\nu_{n}\right) \\
& \quad \leqq C \mathbf{E}\left[h\left(S_{\nu_{n}}\right) ; S_{\nu_{n}}>A_{n} r_{n}, \nu_{n} \leqq \delta_{n} n, \widehat{T}_{g}>\nu_{n}\right] \mathbf{P}\left(T_{0}>n\right) .
\end{aligned}
$$

So it is left to prove that

$$
\mathbf{E}\left[h\left(S_{\nu_{n}}\right) ; S_{\nu_{n}}>A_{n} r_{n}, \nu_{n} \leqq \delta_{n} n, \widehat{T}_{g}>\nu_{n}\right] \rightarrow 0 .
$$

But this immediately follows from (37), since $\widehat{T}_{g} \leqq T_{g}$.

Noting that (45) yields

$$
\mathbf{P}\left(\widehat{T}_{g}>n, \nu_{n} \leqq \delta_{n} n, S_{\nu_{n}}>A_{n} r_{n}, \widehat{T}_{g}>\nu_{n}\right)=o\left(\mathbf{P}\left(T_{0}>n\right)\right),
$$

and taking into account (43), we arrive at the inequality

$$
\limsup _{n \rightarrow \infty} \frac{\mathbf{P}\left(\widehat{T}_{g}>n\right)}{\mathbf{P}\left(T_{0}>n\right)} \leqq \widehat{V}(g) .
$$

Combining (44) and (45), we obtain

$$
\liminf _{n \rightarrow \infty} \frac{\mathbf{P}\left(\widehat{T}_{g}>n\right)}{\mathbf{P}\left(T_{0}>n\right)} \geqq \liminf _{n \rightarrow \infty} \mathbf{E}\left[h\left(S_{\nu_{n}}\right) ; \widehat{T}_{g}>\nu_{n}, \nu_{n} \leqq n \delta_{n}\right] .
$$

It follows from Lemma 2 with $\delta=\delta_{n}$ and $\varepsilon_{n}=C \delta_{n} /(2 \log n)$ that

$$
\mathbf{E}\left[h\left(S_{\delta_{n} n}-g\left(\delta_{n} n\right)\right) ; \widehat{T}_{g}>\delta_{n} n, \nu_{n}>n \delta_{n}\right] \leqq h(c(n)) \mathbf{P}\left(\nu_{n}>n \delta_{n}\right) \rightarrow 0 .
$$

Copyright (c) by SIAM. Unauthorized reproduction of this article is prohibited. 
Consequently,

$$
\mathbf{E}\left[h\left(S_{\nu_{n}}\right) ; \widehat{T}_{g}>\nu_{n}, \nu_{n} \leqq n \delta_{n}\right]=\mathbf{E}\left[h\left(S_{\theta_{n}}-g\left(\theta_{n}\right)\right) ; \widehat{T}_{g}>\theta_{n}\right]+o(1),
$$

where $\theta_{n}:=\nu_{n} \wedge \delta_{n} n$. Applying the optional stopping theorem to the supermartingale $h\left(S_{n}-g(n)\right) 1\left\{\widehat{T}_{g}>n\right\}$, we obtain

$$
\mathbf{E}\left[h\left(S_{\theta_{n}}-g\left(\theta_{n}\right)\right) ; \widehat{T}_{g}>\theta_{n}\right] \geqq \widehat{V}(g) .
$$

Combining this with (46), we complete the proof of Theorem 3.

\section{REFERENCES}

[1] V. I. Afanasyev, J. Geiger, G. Kersting, and V. A. Vatutin, Criticality for branching processes in random environment, Ann. Probab., 33 (2005), pp. 645-673.

[2] F. Aurzada and T. Kramm, The First Passage Time Problem over a Moving Boundary for Asymptotically Stable Lévy Processes, preprint, arXiv:1305.1203, 2015.

[3] F. Aurzada, T. Kramm, and M. Savov, First Passage Times of Lévy Processes over a OneSided Moving Boundary, preprint, arXiv:1201.1118, 2012.

[4] D. Denisov and V. Wachtel, Random walks in cones, Ann. Probab., 43 (2015), pp. 992-1044.

[5] R. A. Doney, Local behaviour of first passage probabilities, Probab. Theory Relat. Fields, 152 (2012), pp. 559-588.

[6] K. B. Erickson, The strong law of large numbers when the mean is undefined, Trans. Amer. Math. Soc., 185 (1973), pp. 371-381.

[7] W. Feller, An Introduction to Probability Theory and Its Applications, Vol. 2, Wiley, New York, 1971.

[8] P. E. Greenwood And I. Monroe, Random stopping preserves regular variation of process distributions, Ann. Probab., 5 (1977), pp. 42-51.

[9] P. E. Greenwood and A. A. Novikov, One-sided boundary crossing for processes with independent increments, Theory Probab. Appl., 31 (1987), pp. 221-232.

[10] B. M. Hambly, G. Kersting, and A. E. Kyprianou, Law of the iterated logarithm for oscillating random walks conditioned to stay nonnegative, Stochastic Process. Appl., 108 (2003), pp. 327-343.

[11] A. A. Mogulskin And E. A. Pecherskit, Time of first entry into a region with curved boundary, Siberian Math. J., 19 (1979), pp. 582-595.

[12] A. A. Novikov, A martingale approach in problems on first crossing time of nonlinear boundaries, Proc. Steklov Inst. Math., 158 (1983), pp. 141-163.

[13] A. A. Novikov, The crossing time of a one-sided nonlinear boundary by sums of independent random variables, Theory Probab. Appl., 27 (1983), pp. 688-702.

[14] K. Uсhiуama, Brownian first exit from and sojourn over one-sided moving boundary and application, Z. Wahrsch. Verw. Geb., 54 (1980), pp. 75-116.

Copyright $@$ by SIAM. Unauthorized reproduction of this article is prohibited. 